\title{
Are seroindeterminate western blot patterns in human T-Cell lymphotropic virus type 1 (Htlv-1) infected individuals associated with low proviral load levels?
}

\author{
Camila Cánepa*, Jimena Salido, Sindy Fraile, Matías Ruggieri, Mirna Biglione, Carolina Berini \\ From 17th International Conference on Human Retroviruses: HTLV and Related Viruses \\ Trois Ilets, Martinique. 18-21 June 2015
}

For HTLV-1/2 diagnosis, reactive screening results are generally confirmed by Western Blot (WB). However, the large number of indeterminate WB patterns is still a problem worldwide. The aim of this study was to determine whether low levels of proviral load (PVL) are associated with seroindeterminate results by WB. PVL was determined in 49 HTLV-1 samples confirmed by $n-P C R$ and classified as G1: positive by WB from individuals with disease ( $\mathrm{n}=27,5$ ATLL and 22 HAM/TSP); G2: positive by WB from asymptomatic carriers $(n=18)$; and $G 3$ : seroindeterminate samples by WB from asymptomatic carriers $(n=4)$. The viral gen pol and albumin were quantified by real-time SYBR Green PCR (ABI Prism SystemAppliedBiosystems 75). Calibration curves were constructed using a DNA stock from MT2 cell line (Limit of quantification: 3 pol copies/reaction-R2>0.99) and the analysis was performed by Krustall Wallis (GraphPad Prism v.5). Median PVL values were 4.03, 1.58 and 0.15 copies of HTLV-1/1 PBMCs fo $\mathrm{r}$ G1, G2 and G3, respectively. Correlation between PVL and age of patients $(\mathrm{S}=0.61)$ was found for $\mathrm{G} 1$ samples. PVL median values were significantly different between the three groups $(\mathrm{p}=$ $0.3)$; the difference was also observed ( $\mathrm{p}=0.5)$ when considering HTLV-1 positive samples by WB [G1 + G2] as a single group. Even though a seroconversion could not be discarded in seroindeterminate cases, a low viral replication rate due to other factors could trigger a weak immune response, thus causing seroindeterminate WB patterns. The present study clearly demonstrates that such cases could be associated to a low HTLV-1 PVL and

Instituto de Investigaciones Biomédicas en Retrovirus y SIDA (INBIRS), UBACONICET, Buenos Aires, Argentina 